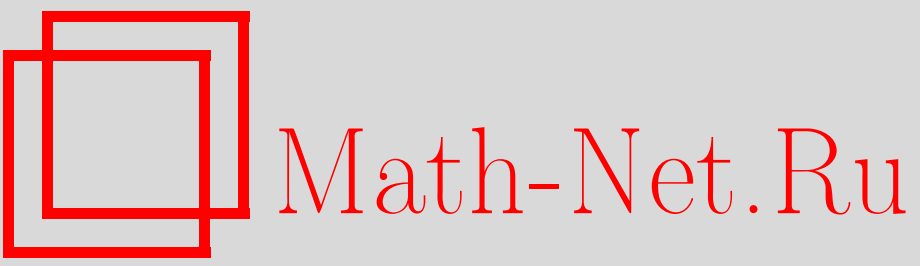

В. Н. Сачков, Случайные отображения с неподвижными элементами, Матем. вопр. криптогр., 2011, том 2, выпуск 2, 95-118

DOI: https://doi.org/10.4213/mvk33

Использование Общероссийского математического портала Math-Net.Ru подразумевает, что вы прочитали и согласны с пользовательским соглашением

http://www.mathnet.ru/rus/agreement

Параметры загрузки:

IP : 54.81 .137 .203

26 апреля 2023 г., 16:51:47 


\author{
МАТЕМАТИЧЕСКИЕ ВОПРОСЫ КРИПТОГРАФИИ \\ 2011 T. 2 № 2 C. 95-118
}

УДК 519.212.2

\title{
Случайные отображения с неподвижными элементами
}

\author{
В. Н. Сачков
}

Академия криптографии Российской Федерации, Москва

Получено 22.IV.2010

Рассматриваются ориентированные графы случайных отображений $n$-элементного множества в себя, выбираемых случайно и равновероятно из множества всех отображений, имеющих неподвижную точку. Найдены точные и предельные (при $n \rightarrow \infty$ ) распределения числа вершин в дереве или лесе, которые содержат заданную вершину, и высоты заданной вершины при условии, что она принадлежит лесу. Найдены также точные и предельные распределения высоты заданной вершины в случайном корневом дереве или лесе с $n$ вершинами.

Ключевые слова: случайные отображения с неподвижными элементами, орграфы, корневые деревья, леса, высота заданной вершины, предельные распределения

\section{Random mappings with fixed elements}

\section{N. Sachkov}

Academy of Cryptography of Russian Federation, Moscow

Abstract. We consider orgraphs of random mappings of $n$-element set having uniform distribution on the set of all mappings with fixed points. Formulas for exact and limit distributions of the number of vertices in a tree or in a forest containing a given vertex are derived along with analogous results for the height of a given vertex conditioned to belong to a forest. Exact and limit distributions of the height of a given vertex in a random rooted tree or forest with $n$ vertices are derived also.

Key words: random mappings with fixed elements, orgraphs, rooted trees, forests, height of a vertex, limit distributions

Citation: Mathematical Aspects of Cryptography, 2011, vol. 2, no. 2, pp. 95-118 (Russian). 


\section{Введение}

Пусть $X$-n-множество и $\sigma_{n}-$ совокупность всех отображений $\sigma: X \rightarrow X$. Будем рассматривать множество отображений $F_{n}=\left\{\sigma \in \sigma_{n}:\right.$ существует $x \in X, \sigma(x)=x\}$. Отображения $\sigma \in F_{n}$ будем называть отображениями с неподвижными элементами. Множество отображений $\sigma_{n} \backslash F_{n}$, не содержащих неподвижных точек, обозначим через $G_{n}$. Орграф, соответствующий отображению $\sigma \in \sigma_{n}$, будем обозначать через $\Gamma(\sigma)$. Отображению $\sigma \in F_{n}$, имеющему единственный циклический элемент (совпадающий с единственным неподвижным элементом), соответствует орграф $\Gamma(\sigma)$, являющийся корневым деревом, дуги которого ориентированы к корню - неподвижному элементу $\sigma$. Такое дерево будем называть изолированным корневым деревом. Любому отображению $\sigma \in F_{n}$ соответствует орграф $\Gamma(\sigma)$, компоненты связности которого являются либо изолированными корневыми деревьями, образующими корневой лес, либо не содержат неподвижных элементов и отвечают связным отображениям из совокупности $G_{m}, 2 \leq m<n$.

Отметим, что отображения из совокупности $F_{n}$ можно рассматривать с использованием понятия А-отображения, определенного в статье [2] и исследованного в монографиях [3] и [4] с использованием производящих и характеристических функций. В настоящей работе рассматривается другой подход, основанный на использовании классических комбинаторных тождеств. Оказывается, что для изучения некоторых характеристик отображений $\sigma \in F_{n}$, представляющих интерес для криптографических исследований, применение таких тождеств является более эффективным, чем использование производящих функций.

Кратко изложим содержание работы.

В § 1 приводится совокупность тождеств, являющихся обобщениями биномиального тождества Абеля [7]. К их числу относятся тождества Коши, Риордана и их модификации. Эти тождества неоднократно используются в дальнейшем изложении, что послужило причиной для выделения их в отдельный параграф. Доказательства основных тождеств можно найти в книге [5]. Производные от них тождества, используемые в работе, доказываются в ее тексте.

В 22 при задании равномерных распределений на множествах $\sigma_{n}$ и $F_{n}$ рассматриваются случайные величины $\eta\left(\sigma_{n}\right)$ и $\eta\left(F_{n}\right)$, равные числу вершин в корневых лесах из изолированных деревьев в орграфах случайных отображений $\sigma \in \sigma_{n}$ и $\sigma \in F_{n}$ соответственно. Для этих случайных вели- 
чин найдены точные и предельные распределения при $n \rightarrow \infty$, а также точные и асимптотические формулы для средних значений. Для множества $\sigma_{n}^{(1)}$ таких отображений $\sigma \in \sigma_{n}$, орграфы которых содержат единственное изолированное дерево, аналогичные результаты получены для случайной величины $\xi^{(1)}$, равной числу вершин в дереве при случайном выборе отображения $\sigma \in \sigma_{n}^{(1)}$. Для случайного отображения $\sigma \in \sigma_{n}$ точное распределение числа вершин изолированного дерева в орграфе $\Gamma(\sigma)$ при условии, что $\Gamma(\sigma)$ имеет такое дерево, отличается от точного распределения $\xi^{(1)}$, однако их предельные распределения и асимптотики для средних при $n \rightarrow \infty$ совпадают.

Для случайных величин $\varsigma\left(\sigma_{n}\right)$ и $\zeta\left(F_{n}\right)$, равных числу изолированных деревьев в орграфе $\Gamma(\sigma)$ при случайном выборе $\sigma \in \sigma_{n}$ и $\sigma \in F_{n}$ соответственно найдены предельные распределения и асимптотики для средних значений при $n \rightarrow \infty$.

В $\S 3$ для случайного отображения $\sigma \in F_{n}$ найдена формула для вероятности того, что заданная вершина принадлежит лесу из корневых изолированных деревьев, являющихся компонентами $\Gamma(\sigma)$. Для этой вероятности приводится асимптотика при $n \rightarrow \infty$.

Для случайного отображения $\sigma \in F_{n}$ найдены точное и предельное распределения случайной величины $\tilde{\eta}_{n}^{(1)}$, равной числу вершин леса, содержащего заданную вершину. Установлено, что случайная величина $\tilde{\eta}_{n}^{(1)} / n$ при $n \rightarrow \infty$ имеет предельное распределение, известное как закон арксинуса [6]. Получены точная и асимптотическая формулы для среднего значения $\tilde{\eta}_{n}^{(1)}$. Аналогичные результаты получены для случайной величины $\tilde{\xi}_{n}$, равной числу вершин изолированного дерева, содержащего заданную вершину и принадлежащего орграфу $\Gamma(\sigma)$ при случайном выборе $\sigma \in F_{n}$.

Для случайной величины $\tilde{\xi}_{n}^{(1)}$, равной числу вершин изолированного дерева, содержащего заданную вершину и принадлежащего случайному корневому лесу с $m$ вершинами, получены точное и предельное распределения, а также точная и асимптотическая формулы для среднего значения при $m \rightarrow \infty$.

В § 4 получены точное и предельное распределения для случайной величины $\chi_{j}^{(1)}$, равной высоте заданной вершины в случайном корневом дере- 
ве с $j$ вершинами. Для случайной величины $\chi_{j}^{(1)} / j$ предельным является распределение Релея. Найдены точная и асимптотическая формулы для среднего значения $\chi_{j}^{(1)}$ при $j \rightarrow \infty$.

Для случайного леса с $m$ вершинами найдены точное и предельное распределения случайной величины $\chi_{m}^{(1)}$, равной высоте заданной вершины в дереве, принадлежащем лесу. Показано, что случайная величина $\chi_{m}^{(1)} / \sqrt{m}$ имеет при $m \rightarrow \infty$ распределение Релея. Получены точная и асимптотическая формулы для среднего значения случайной величины $\chi_{m}^{(1)}$ при $m \rightarrow \infty$.

Для случайного отображения $\sigma \in F_{n}$ получены точное и предельное распределения случайной величины $\tilde{\chi}_{n}$, равной высоте заданной вершины при условии, что она принадлежит лесу в орграфе $\Gamma(\sigma)$. Для случайной величины $\tilde{\chi}_{n} / \sqrt{n}$ предельным распределением является так называемое отраженное нормальное распределение [6].

Для среднего и дисперсии случайной величины $\tilde{\chi}_{n}$ найдены точные и асимптотические формулы при $n \rightarrow \infty$. Дисперсия для $\tilde{\chi}_{n}$ имеет порядок квадрата от среднего значения.

В заключение отметим, что метод с применением классических тождеств может быть использован для рассматриваемых случайных величин при отыскании дисперсий и моментов более высоких порядков.

\section{$\S 1$. Основные формулы}

Для изучения некоторых характеристик случайных отображений $\sigma \in \sigma_{n}$ и $\sigma \in F_{n}$ оказывается эффективным использование тождеств, являющихся обобщениями биномиальной формулы Абеля [7]. Эти тождества связаны с рассмотрением сумм вида

$$
A_{n}(x, y, p, q)=\sum_{k=0}^{n}\left(\begin{array}{l}
n \\
k
\end{array}\right)(x+k)^{k+p}(y+n-k)^{n-k+q} .
$$

Обобщенная формула Абеля имеет вид

$$
A_{n}(x, y,-1,0)=x^{-1}(x+y+n)^{n}, x \neq 0 .
$$

Из равенства (1.1) следует соотношение

$$
A_{n}(x, y, p, q)=x A_{n}(x, y, p-1, q)+n A_{n-1}(x+1, y, p, q),
$$


из него вытекает тождество, которое обычно называют формулой Коши:

$$
A_{n}(x, y, 0,0)=\sum_{k=0}^{n}(n)_{k}(x+y+n)^{n-k} .
$$

Повторное применение соотношения (1.3) приводит к равенству

$$
A_{n}(x, y, p, q)=\sum_{k=0}^{n}(n)_{k}(x+k) A_{n-k}(x+k, y, p-1, q),
$$

из которого следует тождество Риордана

$$
A_{n}(x, y, 1,0)=\sum_{k=0}^{n}(n)_{k}\left[\left(\begin{array}{c}
k+1 \\
2
\end{array}\right)+x(k+1)\right](x+y+n)^{n-k} .
$$

Из соотношения

$$
A_{n}(x, y, p, q)=A_{n-1}(x, y+1, p, q+1)+A_{n-1}(x+1, y, p+1, q)
$$

вытекает тождество

$$
A_{n}(x, y,-1,-1)=\left(x^{-1}+y^{-1}\right)(x+y+n)^{n-1}, x \neq 0, y \neq 0 .
$$

Применение тождеств (1.2) и (1.8) позволяет находить простые выражения для точных распределений характеристик случайных отображений $\sigma \in \sigma_{n}$ и $\sigma \in F_{n}$.

Тождества (1.4) и (1.6) и производные от них с использованием асимптотической формулы [3], [4]

$$
e^{-n} \sum_{k=0}^{n} \frac{n^{k}}{k !}=\frac{1}{2}(1+o(1)), \quad n \rightarrow \infty,
$$

дают возможность получать предельные распределения характеристик и асимптотики для средних значений.

\section{§ 2. Число вершин изолированных деревьев в орграфах отображений}

$1^{0}$. Пусть $X=\{1,2, \ldots, n\}, \sigma_{n}-$ совокупность всех отображений $\sigma: X \rightarrow X$

$$
F_{n}=\left\{\sigma \in \sigma_{n}: \text { существует } x \in X, \sigma(x)=x\right\}
$$

— множество всех отображений с неподвижными элементами. 
Отображению $\sigma$, которое содержит единственный циклический элемент (являющийся неподвижным элементом), соответствует ориентированное корневое дерево, корнем которого является неподвижный элемент. Компоненты связности орграфа $\Gamma(\sigma), \sigma \in F_{n}$, являющиеся деревьями данного вида, будем называть изолированными корневыми деревьями.

Если $G_{n}=\left\{\sigma \in \sigma_{n}: \sigma(x) \neq x\right.$ для каждого $\left.x \in X\right\}$, то для любого $\sigma \in G_{n}$ орграф $\Gamma(\sigma)$ не содержит изолированных деревьев. Отображения из множества $G_{n}$ изучались в статье [1]. Очевидно, что число отображений $\sigma \in G_{n}$ равно $(n-1)^{n}$. Поэтому число отображений $\sigma \in F_{n}$ дается равенством

$$
\left|F_{n}\right|=n^{n}-(n-1)^{n} .
$$

Структура любого отображения $\sigma \in F_{n}$ выглядит следующим образом. Для любого $\sigma \in F_{n}$ существует такое разбиение $X=X_{1} \cup X_{2}$, $X_{1} \cap X_{2}=\varnothing$, что $X_{1} \neq \varnothing$ и компоненты связности орграфа $\Gamma(\sigma)$ являются либо изолированными корневыми деревьями с вершинами, принадлежащими $X_{1}$, либо являются орграфами с вершинами из $X_{2}$, не содержащими неподвижных точек. При этом множество $X_{2}$ может быть пустым, и в этом случае орграф $\Gamma(\sigma)$ для $\sigma \in F_{n}$ является лесом, состоящим из корневых изолированных деревьев с общим числом вершин, равным $n$. В соответствии с известной формулой [4], число лесов с $n$ вершинами равно

$$
R_{n}=(n+1)^{n-1} .
$$

С учетом структуры компонент орграфа $\Gamma(\sigma), \sigma \in F_{n}$, для числа таких отображений, орграф которых имеет корневой лес из изолированных деревьев с общим числом вершин, равным $m$, имеем формулу

$$
M_{n}(m)=\left(\begin{array}{c}
n \\
m
\end{array}\right)(m+1)^{m-1}(n-m-1)^{n-m}, m=1,2, \ldots, n .
$$

Попутно отметим, что

$$
M_{n}(0)=(n-1)^{n},
$$

так как $M_{n}(0)=\left|G_{n}\right|$.

На множествах $\sigma_{n}$ и $F_{n}$ зададим равномерные вероятностные распределения и рассмотрим случайные величины $\eta\left(\sigma_{n}\right)$ и $\eta\left(F_{n}\right)$, равные числу вершин в корневых лесах из изолированных деревьев в орграфах отображе- 
ний $\sigma \in \sigma_{n}$ и $\sigma \in F_{n}$ соответственно. Распределения этих случайных величин имеют вид

$$
\begin{aligned}
& \mathbf{P}\left(\eta\left(\sigma_{n}\right)=m\right)=\frac{1}{n^{n}} M_{n}(m), m=0,1, \ldots, n, \\
& \mathbf{P}\left(\eta\left(F_{n}\right)=m\right)=\frac{1}{n^{n}-(n-1)^{n}} M_{n}(m), m=1,2, \ldots, n .
\end{aligned}
$$

При $m=o(n)$ и $n \rightarrow \infty$ для этих распределений имеем асимптотические представления

$$
\begin{aligned}
& \mathbf{P}\left(\eta\left(\sigma_{n}\right)=m\right)=\frac{(m+1)^{m-1}}{e^{m+1} m !}(1+o(1)), m=0,1, \ldots, \\
& \mathbf{P}\left(\eta\left(F_{n}\right)=m\right)=\frac{1}{1-e^{-1}} \frac{(m+1)^{m-1}}{e^{m+1} m !}(1+o(1)), m=1,2, \ldots .
\end{aligned}
$$

Очевидно, что средние значения $\eta\left(\sigma_{n}\right)$ и $\eta\left(F_{n}\right)$ выражаются через одну и ту же величину

$$
\tilde{M}_{n}=\sum_{m=1}^{n} m\left(\begin{array}{l}
n \\
m
\end{array}\right)(m+1)^{m-1}(n-m-1)^{n-m},
$$

а именно:

$$
\mathbf{M} \eta\left(\sigma_{n}\right)=\frac{1}{n^{n}} \tilde{M}_{n}, \mathbf{M} \eta\left(F_{n}\right)=\frac{1}{n^{n}-(n-1)^{n}} \tilde{M}_{n} .
$$

Проводя замену переменной, получаем, что

$$
\tilde{M}_{n}=n \sum_{m=0}^{n-1}\left(\begin{array}{c}
n-1 \\
m
\end{array}\right)(m+2)^{m}(n-1-m-1)^{n-1-m} .
$$

Применение тождества Коши (1.4) приводит к равенствам

$$
\tilde{M}_{n}=n A_{n-1}(2,-1,0,0)=n \sum_{m=0}^{n-1}(n-1)_{m} n^{n-m-1},
$$

из которых следует, что

$$
\tilde{M}_{n}=n ! \sum_{m=0}^{n-1} \frac{n^{m}}{m !}
$$


Теперь из формул (2.8) и (2.9) при $n \rightarrow \infty$, применяя формулу Стирлинга, получаем асимптотические представления

$$
\mathbf{M} \eta\left(\sigma_{n}\right)=\sqrt{\frac{\pi n}{2}}(1+o(1)), \quad \mathbf{M} \eta\left(F_{n}\right)=\frac{1}{1-e^{-1}} \sqrt{\frac{\pi n}{2}}(1+o(1)) .
$$

$2^{0}$. Пусть $\sigma_{n}^{(1)}-$ совокупность всех отображений $\sigma \in \sigma_{n}$, орграфы которых содержат единственное изолированное дерево. Число рассматриваемых отображений в $\sigma_{n}^{(1)}$ равно числу отображений $\sigma \in \sigma_{n}$, имеющих одну неподвижную точку и, следовательно, имеем

$$
\left|\sigma_{n}^{(1)}\right|=n(n-1)^{n-1} .
$$

Рассмотрим случайную величину $\xi^{(1)}$, равную числу вершин изолированного дерева в орграфе $\Gamma(\sigma)$ случайно выбранного отображения $\sigma \in \sigma^{(1)}$. Распределение $\xi^{(1)}$ имеет вид

$$
\mathbf{P}\left(\xi^{(1)}=j\right)=\frac{1}{n(n-1)^{n-1}}\left(\begin{array}{c}
n \\
j
\end{array}\right) j^{j-1}(n-j-1)^{n-j}, \quad j=1,2, \ldots, n .
$$

При $j=o(n)$ и $n \rightarrow \infty$ отсюда получаем, что

$$
\mathbf{P}\left(\xi^{(1)}=j\right)=\frac{j^{j-1}}{e^{j} j !}(1+o(1)), \quad j=1,2, \ldots
$$

Для среднего значения случайной величины $\xi^{(1)}$ из равенства (2.12) следует формула

$$
\mathbf{M} \xi^{(1)}=\frac{1}{n(n-1)^{n-1}} \sum_{j=1}^{n}\left(\begin{array}{l}
n \\
j
\end{array}\right) j^{j}(n-j-1)^{n-j},
$$

из которой следует, что

$$
\mathbf{M} \xi^{(1)}=\frac{1}{(n-1)^{n-1}} \sum_{j=0}^{n-1}\left(\begin{array}{c}
n-1 \\
j
\end{array}\right)(j+1)^{j}(n-1-j-1)^{n-j-1} .
$$

Применяя формулу Коши (1.4), получаем

$$
\mathbf{M} \xi^{(1)}=\frac{1}{(n-1)^{n-1}} A_{n-1}(1,-1,0,0)=\frac{1}{(n-1)^{n-1}} \sum_{k=0}^{n-1}(n-1)_{k}(n-1)^{n-k-1} .
$$

Отсюда следует, что

$$
\mathbf{M} \xi^{(1)}=\frac{(n-1) !}{(n-1)^{n-1}} \sum_{m=0}^{n-1} \frac{(n-1)^{m}}{m !} .
$$


Используя формулу (1.9) и формулу Стирлинга при $n \rightarrow \infty$, получаем асимптотику

$$
\mathbf{M} \xi^{(1)}=\sqrt{\frac{\pi n}{2}}(1+o(1)) .
$$

$3^{0}$. Известно [4], что если $\varsigma_{n}^{(1)}-$ число деревьев в случайном корневом лесе с $m$ помеченными вершинами, то

$$
\mathbf{P}\left(\varsigma_{m}^{(1)}=k\right)=\left(\begin{array}{c}
m-1 \\
k-1
\end{array}\right) \frac{m^{m-k}}{(m+1)^{m-1}}, k=1,2, \ldots, m,
$$

и при $m \rightarrow \infty$ случайная величина $\varsigma_{m}^{(1)}-1$ имеет в пределе распределение Пуассона с параметром $\lambda=1$, т. е.

$$
\mathbf{P}\left(\varsigma_{m}^{(1)}-1=r\right) \rightarrow \frac{e^{-1}}{r !}, \quad r=0,1, \ldots
$$

Сравним теперь с этими результатами асимптотические распределения случайных величин $\varsigma\left(\sigma_{n}\right)$ и $\varsigma\left(F_{n}\right)$, равных числам изолированных корневых деревьев в орграфе $\Gamma(\sigma)$ для случайно выбранных отображений $\sigma \in \sigma_{n}$ и $\sigma \in F_{n}$ соответственно.

Случайные величины $\varsigma\left(\sigma_{n}\right)$ и $\varsigma\left(F_{n}\right)$ имеют, соответственно, биномиальное распределение

$$
\mathbf{P}\left(\varsigma\left(\sigma_{n}\right)=k\right)=\frac{1}{n^{n}}\left(\begin{array}{l}
n \\
k
\end{array}\right)(n-1)^{n-k}, k=0,1, \ldots, n,
$$

и усеченное биномиальное распределение

$$
\mathbf{P}\left(\varsigma\left(F_{n}\right)=k\right)=\frac{1}{n^{n}-(n-1)^{n}}\left(\begin{array}{l}
n \\
k
\end{array}\right)(n-1)^{n-k}, k=1,2, \ldots, n .
$$

Производящие функции этих распределений имеют, соответственно, вид

$$
\psi_{n}(t)=\frac{1}{n^{n}}(n+t-1)^{n}, \quad \tilde{\psi}_{n}(t)=\frac{(n+t-1)^{n}-(n-1)^{n}}{n^{n}-(n-1)^{n}} .
$$

Поэтому при $n \rightarrow \infty$ для любого $t$ имеем

$$
\psi_{n}(t) \rightarrow e^{t-1}, \quad \tilde{\psi}_{n}(t) \rightarrow \frac{e^{t}-1}{e-1} .
$$

Следовательно,

$$
\begin{aligned}
& \mathbf{P}\left(\varsigma\left(\sigma_{n}\right)=k\right) \rightarrow \frac{1}{e} \frac{1}{k !}, \quad k=0,1, \ldots, \\
& \mathbf{P}\left(\varsigma\left(F_{n}\right)=k\right) \rightarrow \frac{1}{e-1} \frac{1}{k !}, \quad k=1,2, \ldots .
\end{aligned}
$$


Отметим, что при $n \rightarrow \infty$ асимптотические формулы для математических ожиданий $\varsigma\left(\sigma_{n}\right)$ и $\varsigma\left(F_{n}\right)$ имеют вид

$$
\begin{aligned}
& \mathbf{M} \varsigma\left(\sigma_{n}\right) \rightarrow 1, \\
& \mathbf{M}_{\zeta}\left(F_{n}\right) \rightarrow \frac{e}{e-1},
\end{aligned}
$$

тогда как в соответствии с (2.17) при $m \rightarrow \infty$

$$
\mathbf{M} \varsigma_{m}^{(1)} \rightarrow 2
$$

\section{§ 3. Леса и деревья с заданной вершиной в орграфах отображений}

$1^{0}$. Обозначим через $V_{n}$ число преобразований $\sigma \in F_{n}$, для которых оргpaф $\Gamma(\sigma)$ имеет лес из корневых изолированных деревьев, содержащий заданную вершину $x \in X$. Число способов выбора таких лесов с $m$ вершинами в силу (2.2) равно $\left(\begin{array}{c}n-1 \\ m-1\end{array}\right)(m+1)^{m-1}$. Остальные компоненты связности $\Gamma(\sigma)$ соответствуют некоторому отображению из $G_{n-m}$, и число способов построения отвечающей ему части орграфа $Г(\sigma)$ равно $(n-m-1)^{n-m}$. Следовательно,

$$
V_{n}=\sum_{m=1}^{n}\left(\begin{array}{c}
n-1 \\
m-1
\end{array}\right)(m+1)^{m-1}(n-m-1)^{n-m}=\sum_{m=0}^{n-1}\left(\begin{array}{c}
n-1 \\
m
\end{array}\right)(m+2)^{m}(n-m-2)^{n-m-1} .
$$

Используя тождество Коши (1.4), получаем

$$
V_{n}=A_{n-1}(2,-1,0,0)=(n-1) ! \sum_{m=0}^{n-1} \frac{n^{m}}{m !} .
$$

При $n \rightarrow \infty$, применяя формулу (1.9), имеем

$$
V_{n}=n^{n} \sqrt{\frac{\pi}{2 n}}(1+o(1)) .
$$

Для случайно равновероятно выбранного отображения $\sigma \in F_{n}$ обозначим через $P_{n}$ вероятность того, что лес из изолированных корневых деревьев в орграфе $\Gamma(\sigma)$ содержит заданную вершину $x \in X$. Эта вероятность совпадает с вероятностью того, что случайно выбранная вершина из множества $X$ в орграфе $\Gamma(\sigma), \sigma \in F_{n}$, окажется принадлежащей лесу.

Из определения вероятности $P_{n}$ следует, что

$$
P_{n}=\frac{V_{n}}{n^{n}-(n-1)^{n}},
$$


и, следовательно, из равенства (3.1) получаем формулу

$$
P_{n}=\frac{1}{n^{n}-(n-1)^{n}} \sum_{m=1}^{n}\left(\begin{array}{c}
n-1 \\
m-1
\end{array}\right)(m+1)^{m-1}(n-m-1)^{n-m} .
$$

При $n \rightarrow \infty$ из равенств (3.3) и (3.4) следует асимптотическая формула

$$
P_{n}=\frac{e}{e-1} \sqrt{\frac{\pi}{2 n}}(1+o(1))
$$

2. ${ }^{0}$. Рассмотрим подмножество отображений $F_{n}^{\prime} \subseteq F_{n}$ такое, что лес орграфа $\Gamma(\sigma)$ при $\sigma \in F_{n}^{\prime}$ содержит заданную вершину $x \in X$. Для случайного отображения $\sigma \in F_{n}^{\prime}$ обозначим через $\tilde{\eta}_{n}^{(1)}$ случайную величину, равную числу вершин леса в $\Gamma(\sigma)$, содержащего вершину $x \in X$. Вероятностное распределение $\tilde{\eta}_{n}^{(1)}$ имеет вид

$$
\mathbf{P}\left(\tilde{\eta}_{n}^{(1)}=m\right)=\frac{1}{V_{n}}\left(\begin{array}{c}
n-1 \\
m-1
\end{array}\right)(m+1)^{m-1}(n-m-1)^{n-m}, m=1,2, \ldots, n,
$$

где $V_{n}$ определяется равенством (3.1).

Из формул (3.3) и (3.6) с использованием формулы Стирлинга получаем предельную теорему.

Теорема 1. Предельные распределения $\tilde{\eta}_{n}^{(1)}$ имеют следующий вид:

1) если $n \rightarrow \infty$, то

$$
\mathbf{P}\left(\tilde{\eta}_{n}^{(1)}=m\right)=\sqrt{\frac{2}{\pi}} \frac{(m+1)^{m-1}}{e^{m+1}(m-1) !} \frac{1}{\sqrt{n}}(1+o(1)), m=1,2, \ldots ;
$$

2) если $n, m, n-m \rightarrow \infty$, то

а) для любых $0<\delta_{1}<\delta_{2}<1$ равномерно для всех $x=\frac{n}{m} \in\left[\delta_{1}, \delta_{2}\right]$

$$
\mathbf{P}\left(\frac{\tilde{\eta}_{n}^{(1)}}{n}=x\right)=\frac{1}{\pi} \frac{1}{\sqrt{x(1-x)}} \frac{1}{n}(1+o(1)) ;
$$

б) равномерно для всех $0<\alpha<\beta<1$

$$
\mathbf{P}\left(\alpha \leq \frac{\tilde{\eta}_{n}^{(1)}}{n} \leq \beta\right)=\frac{1}{\pi}[\arcsin (2 \beta-1)-\arcsin (2 \alpha-1)](1+o(1)) .
$$


Пункт б) теоремы вытекает из пункта а) и равенства

$$
\int_{\alpha}^{\beta} \frac{d x}{\sqrt{x(1-x)}}=\int_{\alpha-\frac{1}{2}}^{\beta-\frac{1}{2}} \frac{d z}{\sqrt{\frac{1}{4}-z^{2}}} .
$$

Предельное распределение для $\frac{\tilde{\eta}_{n}^{(1)}}{n}$ в теореме 1 является известным законом арксинуса [6]. Из вида распределения (3.8) следует, что наиболее вероятными являются значения $m$ - числа вершин леса, содержащего фиксированную вершину, когда с ростом $n$ величина $x=m / n$ приближается либо к 0 , либо к 1 .

В соответствии с формулой (3.6) среднее значение случайной величины $\tilde{\eta}_{n}^{(1)}$ определяется равенством

$$
\mathbf{M} \tilde{\eta}_{n}^{(1)}=M_{n}^{(1)} / V_{n},
$$

где $V_{n}$ определяется равенством (3.2) и

$$
M_{n}^{(1)}=\sum_{m=1}^{n} m\left(\begin{array}{c}
n-1 \\
m-1
\end{array}\right)(m+1)^{m-1}(n-m-1)^{n-m} .
$$

Представим $M_{n}^{(1)}$ в виде разности

$$
\begin{gathered}
M_{n}^{(1)}=A-B \\
A=\sum_{m=1}^{n}\left(\begin{array}{c}
n-1 \\
m-1
\end{array}\right)(m+1)^{m}(n-m-1)^{n-m}, B=\sum_{m=1}^{n}\left(\begin{array}{c}
n-1 \\
m-1
\end{array}\right)(m+1)^{m-1}(n-m-1)^{n-m} .
\end{gathered}
$$

Преобразуем сумму $A$ :

$$
A=\sum_{m=0}^{n-1}\left(\begin{array}{c}
n-1 \\
m
\end{array}\right)(m+2)^{m+1}(n-m-2)^{n-m-1}=A_{n-1}(2,-1,1,0) .
$$

Применяя тождество Риордана (1.6), получаем

$$
A=\sum_{k=0}^{n-1}\left[\left(\begin{array}{c}
k+1 \\
2
\end{array}\right)+2(k+1)\right](n-1)_{k} n^{n-k-1}=\frac{(n-1) !}{2} \sum_{k=0}^{n-1} \frac{n^{k}}{k !}(n-k)(n-k+3) .
$$


Аналогичными преобразованиями сумму $B$ приводим к виду

$$
B=\sum_{m=0}^{n-1}\left(\begin{array}{c}
n-1 \\
m
\end{array}\right)(m+2)^{m}(n-m-2)^{n-m-1}=A_{n-1}(2,-1,0,0) .
$$

Применяя тождество Коши (1.4), имеем

$$
B=(n-1) ! \sum_{k=0}^{n-1} \frac{n^{k}}{k !}
$$

Теперь из равенств (3.11), (3.13) и (3.14) получаем

$$
M_{n}^{(1)}=\frac{(n-1) !}{2} \sum_{k=0}^{n-1} \frac{n^{k}}{k !}\left[\left(n^{2}+3 n-2\right)-2(n+1) k+k(k-1)\right] .
$$

Это равенство позволяет получить следующее представление для $M_{n}^{(1)}$ :

$$
M_{n}^{(1)}=\frac{(n-1) !}{2}\left(n^{2}+3 n-2\right) \sum_{k=0}^{n-1} \frac{n^{k}}{k !}-(n+1) n ! \sum_{k=0}^{n-2} \frac{n^{k}}{k !}+\frac{n n !}{2} \sum_{k=0}^{n-3} \frac{n^{k}}{k !} .
$$

Отсюда следует, что

$$
M_{n}^{(1)}=\left[\frac{n !}{2}-(n-1) !\right] \sum_{k=0}^{n-1} \frac{n^{k}}{k !}+\frac{3}{2} n^{n} .
$$

Из равенств (3.2), (3.9) и (3.17) имеем

$$
\mathbf{M} \tilde{\eta}_{n}^{(1)}=\frac{n}{2}-1+\frac{3}{2} \frac{n^{n}}{V_{n}}
$$

При $n \rightarrow \infty$, учитывая (3.3), получаем асимптотическую формулу для среднего значения случайной величины $\tilde{\eta}_{n}^{(1)}$ :

$$
\mathbf{M} \tilde{\eta}_{n}^{(1)}=\frac{n}{2}+3 \sqrt{\frac{n}{2 \pi}}(1+o(1))
$$

$3^{0}$. Пусть $F_{n}^{\prime}-$ множество отображений $\sigma \in F_{n}$, для которых заданная вершина $x \in X$ принадлежит корневому лесу орграфа $\Gamma(\sigma)$. Для случайного 
отображения $\sigma \in F_{n}^{\prime}$ обозначим через $\tilde{\xi}_{n}$ случайную величину, равную числу вершин изолированного корневого дерева, содержащего вершину $x \in X$.

Так как число способов построения корневого дерева, содержащего $j$ вершин, включая вершину $x$, равно $\left(\begin{array}{c}n-1 \\ j-1\end{array}\right) j^{j-1}$, а отображение множества остальных $n-j$ элементов на себя произвольно, то распределение $\tilde{\xi}_{n}$ имеет вид

$$
\mathbf{P}\left(\tilde{\xi}_{n}=j\right)=\frac{1}{V_{n}}\left(\begin{array}{c}
n-1 \\
j-1
\end{array}\right) j^{j-1}(n-j)^{n-j}, j=1,2, \ldots, n,
$$

где $V_{n}$ определяется формулой (3.1). Из формул (3.3) и (3.20) следует предельная теорема, аналогичная Теореме 1.

Теорема 2. Предельные распределения $\tilde{\xi}_{n}$ имеют следующий вид:

1) если $n \rightarrow \infty$, то

$$
\mathbf{P}\left(\tilde{\xi}_{n}=j\right)=\sqrt{\frac{2}{\pi}} \frac{j^{j-1}}{e^{j}(j-1) !} \frac{1}{\sqrt{n}}(1+o(1)), \quad j=1,2, \ldots
$$

2) если $n, j, n-j \rightarrow \infty$, mо

а) для любых $0<\delta_{1}<\delta_{2}<1$ равномерно для всех $x=\frac{j}{n} \in\left[\delta_{1}, \delta_{2}\right]$

$$
\mathbf{P}\left(\frac{\tilde{\xi}_{n}}{n}=x\right)=\frac{1}{\pi} \frac{1}{\sqrt{x(1-x)}} \frac{1}{n}(1+o(1)),
$$

б) равномерно для всех $0<\alpha, \beta<1$

$$
\mathbf{P}\left(\alpha \leq \frac{\tilde{\xi}_{n}}{n} \leq \beta\right)=\frac{1}{\pi}[\arcsin (2 \beta-1)-\arcsin (2 \alpha-1)](1+o(1)) .
$$

Доказательство теоремы 2 аналогично доказательству теоремы 1. Поэтому оно не приводится.

Из (3.20) следует, что среднее значение случайной величины $\tilde{\xi}_{n}$ имеет вид

$$
\mathbf{M} \tilde{\xi}_{n}=\frac{W_{n}}{V_{n}},
$$


где

$$
W_{n}=\sum_{j=1}^{n}\left(\begin{array}{c}
n-1 \\
j-1
\end{array}\right) j^{j}(n-j)^{n-j}
$$

Так как

$$
W_{n}=\sum_{j=0}^{n-1}\left(\begin{array}{c}
n-1 \\
j
\end{array}\right)(j+1)^{j+1}(n-j-1)^{n-j-1}=A_{n-1}(1,0,1,0)
$$

то, применяя тождество Риордана (1.6), получаем

$$
W_{n}=\sum_{k=0}^{n-1}(n-1)_{k}\left[\left(\begin{array}{c}
k+1 \\
2
\end{array}\right)+k+1\right] n^{n-k-1}=\frac{(n-1) !}{2} \sum_{j=0}^{n-1} \frac{n^{j}}{j !}(n-j)(n-j+1) .
$$

Представим $W_{n}$ в виде

$$
W_{n}=Q-R+S
$$

где

$$
Q_{n}=\frac{(n+1) !}{2} \sum_{j=0}^{n-1} \frac{n^{j}}{j !}, \quad R_{n}=n ! \sum_{j=0}^{n-1} \frac{n^{j}}{j !} j, \quad S_{n}=\frac{(n-1) !}{2} \sum_{j=0}^{n-1} \frac{n^{j}}{j !} j(j-1)
$$

После проведения преобразований сумм $Q, R$ и $S$ с использованием равенства (3.25) получаем

$$
W_{n}=\frac{n !}{2} \sum_{j=1}^{n-1} \frac{n^{j}}{j !}+\frac{n^{n}}{2}
$$

Из равенств (3.2), (3.23) и (3.26) следует, что

$$
\mathbf{M} \tilde{\xi}_{n}=\frac{n}{2}+\frac{n^{n}}{2(n-1) ! \sum_{j=0}^{n-1} \frac{n^{j}}{j !}}
$$


При $n \rightarrow \infty$ с учетом формулы (3.3) из равенства (3.27) получаем асимптотику

$$
\mathbf{M} \tilde{\xi}_{n}=\frac{n}{2}+\sqrt{\frac{n}{2 \pi}}(1+o(1))
$$

$4^{0}$. Для случайно выбранного корневого леса с $m$ вершинами обозначим через $\tilde{\xi}_{m}^{(1)}$ случайную величину, равную числу вершин дерева, содержащего заданную вершину. Число способов выбора дерева с $j$ вершинами, содержащего заданную вершину, равно $\left(\begin{array}{c}m-1 \\ j-1\end{array}\right) j^{j-1}$, поэтому распределение $\tilde{\xi}_{m}^{(1)}$ с учетом (2.2) имеет вид

$$
\mathbf{P}\left(\tilde{\xi}_{m}^{(1)}=j\right)=\frac{1}{(m+1)^{m-1}}\left(\begin{array}{c}
m-1 \\
j-1
\end{array}\right) j^{j-1}(m-j+1)^{m-j-1}, j=1,2, \ldots, m .
$$

Среднее значение случайной величины $\tilde{\xi}_{m}^{(1)}$ определяется формулой

$$
\mathbf{M} \tilde{\xi}_{m}^{(1)}=\frac{1}{(m+1)^{m-1}} Q_{m}
$$

где

$$
Q_{m}=\sum_{j=1}^{m}\left(\begin{array}{c}
m-1 \\
j-1
\end{array}\right) j^{j}(m-j+1)^{m-j-1}=\sum_{j=0}^{m-1}\left(\begin{array}{c}
m-1 \\
j
\end{array}\right)(j+1)^{j+1}(m-j)^{m-j-2} .
$$

Представим $Q_{m}$ в виде разности

$$
Q_{m}=Q_{m}^{(1)}-Q_{m}^{(2)},
$$

где

$$
\begin{aligned}
& Q_{m}^{(1)}=\sum_{j=0}^{m-1}\left(\begin{array}{c}
m-1 \\
j
\end{array}\right)(j+1)^{j+1}(m-j)^{m-j-1}, \\
& Q_{m}^{(2)}=(m-1) \sum_{j=0}^{m-2}\left(\begin{array}{c}
m-2 \\
j
\end{array}\right)(j+1)^{j+1}(m-j)^{m-j-2} .
\end{aligned}
$$

Отсюда следуют очевидные равенства

$$
Q_{m}^{(1)}=A_{m-1}(1,1,1,0), \quad Q_{m}^{(2)}=(m-1) A_{m-2}(1,2,1,0) .
$$


Применяя тождества Риордана (1.6), получаем

$$
\begin{aligned}
& Q_{m}^{(1)}=\sum_{k=0}^{m-1}(m-1)_{k}\left[\left(\begin{array}{c}
k+1 \\
2
\end{array}\right)+(k+1)\right](m+1)^{m-k-1}, \\
& Q_{m}^{(2)}=(m-1) \sum_{k=0}^{m-2}(m-2)_{k}\left[\left(\begin{array}{c}
k+1 \\
2
\end{array}\right)+(k+1)\right](m+1)^{m-k-2} .
\end{aligned}
$$

После замены переменных суммирования имеем

$$
\begin{aligned}
& Q_{m}^{(1)}=\frac{(m-1) !}{2} \sum_{k=0}^{m-1} \frac{(m+1)^{k}}{k !}(m-k)(m-k+1), \\
& Q_{m}^{(2)}=\frac{(m-1) !}{2} \sum_{k=0}^{m-2} \frac{(m+1)^{k}}{k !}(m-k)(m-k-1) .
\end{aligned}
$$

Теперь из равенств (3.31), (3.32) и (3.33) получаем

$$
Q_{m}=(m+1)^{m-1}+(m-1) ! \sum_{k=0}^{m-2} \frac{(m+1)^{k}}{k !}(m-k) .
$$

Из этого равенства следует, что

$$
Q_{m}=(m+1)^{m-1}+m(m-1)(m+1)^{m-2}-(m-1) ! \sum_{k=0}^{m-3} \frac{(m+1)^{k}}{k !} .
$$

Отсюда получаем представление $Q_{m}$, удобное для асимптотических оценок:

$$
Q_{m}=(m+1)^{m}\left(1+\frac{2}{m}\right)-(m-1) ! \sum_{k=0}^{m+1} \frac{(m+1)^{k}}{k !} .
$$

При $m \rightarrow \infty$ из равенств (3.30), (3.34), формулы (1.9) и формулы Стирлинга следует соотношение

$$
\mathbf{M} \tilde{\xi}_{m}^{(1)}=m-\sqrt{\frac{\pi m}{2}}(1+o(1)) .
$$

\section{§ 4. Высота заданной вершины}

$1^{0}$. Пусть в корневом дереве все дуги ориентированы по направлению к корню. Тогда высота заданной вершины дерева определяется как число 
дуг в ориентированном пути от этой вершины до корня. На множестве из $j^{j-1}$ корневых деревьев с $j$ вершинами зададим равномерное вероятностное распределение и рассмотрим случайную величину $\chi_{j}^{(1)}$, равную высоте заданной вершины в случайно выбранном дереве.

Покажем, что вероятностное распределение случайной величины $\chi_{j}^{(1)}$ имеет вид

$$
\mathbf{P}\left(\chi_{j}^{(1)}=k\right)=\frac{(k+1)(j-1)_{k}}{j^{k+1}}, \quad k=0,1, \ldots, j-1 .
$$

Действительно, имеется $(j-1)_{k}$ способов выбора вершин, входящих в путь длины $k$, включая корневую вершину. Для построения соответствующего дерева выбирается корневой лес, корнями которого являются выбранные $k+1$ вершин, остальные $j-k-1$ вершин являются некорневыми.

Число способов выбора такого леса равно

$$
W(j, k)=\sum_{\substack{i_{1}+\ldots+i_{k+1}=j-k-1 \\ i_{j} \geq 0}} \frac{(j-k-1) !}{i_{1} ! i_{2} ! \ldots i_{k+1} !}\left(i_{1}+1\right)^{i_{1}-1} \ldots\left(i_{k+1}+1\right)^{i_{k+1}-1} .
$$

После замены переменных суммирования отсюда получаем

$$
W(j, k)=(j-k-1) ! \sum_{\substack{i_{1}+\ldots+i_{k+1}=j \\ i_{s}>0}} \frac{i_{1}^{i_{1}-1} \ldots i_{k+1}^{i_{k+1}-1}}{i_{1} ! \ldots . i_{k+1} !} .
$$

Используя известное тождество из книги [4]

$$
\sum_{\substack{i_{1}+\ldots+i_{k}=j \\ i_{s}>0}} \frac{i_{1}^{i_{1}-1} \ldots i_{k}^{i_{k}-1}}{i_{1} ! \ldots . i_{k} !}=\frac{(k+1) j^{j-k-2}}{(j-k-1) !}
$$

находим, что

$$
W(j, k)=(k+1) j^{j-k-2} .
$$

Таким образом, число корневых деревьев с $j$ вершинами, у которых заданная вершина имеет высоту $k$, равно

$$
R(j, k)=(k+1) j^{j-k-2}(j-1)_{k}, \quad k=0, \ldots, j-1 .
$$


Теперь формула (4.1) следует из равенства

$$
\mathbf{P}\left(\chi_{j}^{(1)}=k\right)=\frac{R(j, k)}{j^{j-1}} .
$$

Из формулы (4.1) вытекает следующая теорема.

Теорема 3. Если $j \rightarrow \infty$, то случайная величина $\chi_{j}^{(1)} / \sqrt{j}$ в качестве предельного имеет распределение Релея, а именно:

$$
\begin{gathered}
\mathbf{P}\left(\frac{\chi_{j}^{(1)}}{\sqrt{j}}=x\right)=x e^{-\frac{x^{2}}{2}} \frac{1}{\sqrt{j}}(1+o(1)) \text { равномерно по } x=\frac{k}{\sqrt{j}}=o\left(j^{1 / 6}\right), \\
\mathbf{P}\left(\alpha \leq \frac{\chi_{j}^{(1)}}{\sqrt{j}} \leq \beta\right)=\left[e^{-\frac{\alpha^{2}}{2}}-e^{-\frac{\beta^{2}}{2}}\right](1+o(1)) \text { равномерно по } 0<\alpha<\beta=o\left(j^{1 / 6}\right) .
\end{gathered}
$$

Формула (4.2) получается из (4.1) с помощью формулы Стирлинга, а формула (4.3) выводится из (4.2) интегрированием.

Среднее значение случайной величины $\chi_{j}^{(1)}$ определяется формулой

$$
\mathbf{M} \chi_{j}^{(1)}=\sum_{k=1}^{j-1} \frac{k(k+1)(j-1)_{k}}{j^{k+1}},
$$

из которой следует, что

$$
\mathbf{M} \chi_{j}^{(1)}=\frac{(j-1) !}{j^{j}} \sum_{k=0}^{j-2} \frac{j^{k}}{k !}[j(j-1)-2(j-1) k+k(k-1)] .
$$

Проводя преобразования, аналогичные использованным при выводе из равенства (3.15) соотношения (3.17), получаем

$$
\mathbf{M} \chi_{j}^{(1)}=\frac{j !}{j^{j}} \sum_{k=0}^{j-2} \frac{j^{k}}{k !} .
$$

Отсюда при $j \rightarrow \infty$ имеем асимптотику

$$
\mathbf{M} \chi_{j}^{(1)}=\sqrt{\frac{\pi j}{2}}(1+o(1)) .
$$


$2^{0}$. Рассмотрим теперь случайную величину $\chi_{m}$, равную высоте заданной вершины, принадлежащей случайному лесу из $m$ вершин. Распределение $\chi_{m}$ имеет вид

$$
\mathbf{P}\left(\chi_{m}=k\right)=\frac{k+1}{(m+1)^{m-1}} \sum_{j=k+1}^{m}\left(\begin{array}{c}
m-1 \\
j-1
\end{array}\right)(m-j+1)^{m-j-1}(j-1)_{k} j^{j-k-2}, k=0, \ldots, m-1 .
$$

Действительно, вероятность того, что заданная вершина принадлежит корневому дереву с $j$ вершинами в лесе, имеющем $m$ вершин, равна

$$
\frac{1}{(m+1)^{m-1}}\left(\begin{array}{c}
m-1 \\
j-1
\end{array}\right) j^{j-1}(m-j+1)^{m-j-1} .
$$

Умножив эту вероятность на вероятность того, что заданная вершина имеет высоту $k$ в дереве с $j$ вершинами, с учетом формулы (4.1), после суммирования полученных произведений придем к (4.6).

Из равенства (4.6) получаем следующее выражение для распределения $\chi_{m}$ :

$$
\mathbf{P}\left(\chi_{m}=k\right)=\frac{(k+1)(m-1)_{k}}{(m+1)^{m-1}} E(m, k)
$$

где

$$
E(m, k)=\sum_{j=0}^{m-k-1}\left(\begin{array}{c}
m-k-1 \\
j
\end{array}\right)(j+k+1)^{j-1}(m-j-k)^{m-j-k-2} .
$$

К сумме $E(m, k)$ применим тождество (1.8), выведенное в § 1 .

В результате получаем

$$
E(m, k)=A_{m-k-1}(k+1,1,-1,-1)=\frac{(k+1)}{(k+1)}(m+1)^{m-k-2} .
$$

Теперь из формул (4.7) и (4.8) вытекает окончательный вид распределения $\chi_{m}$ :

$$
\mathbf{P}\left(\chi_{m}=k\right)=\frac{(k+2)(m-1)_{k}}{(m+1)^{k+1}}, \quad k=0,1, \ldots, m-1 .
$$


Действительно, из формулы (4.9) следует, что при $m \rightarrow \infty$ и $x=o\left(m^{1 / 6}\right)$

$$
\mathbf{P}\left(\frac{\chi_{m}}{\sqrt{m}}=x\right)=x e^{-\frac{x^{2}}{2}} \frac{1}{\sqrt{m}}(1+o(1))
$$

Это означает, что случайная величина $\chi_{m} / \sqrt{m}$ в пределе имеет распределение Релея и что для этой случайной величины справедлива теорема, аналогичная теореме 3 , если заменить в ней $\chi_{j}^{(1)} / \sqrt{j}$ на $\chi_{m} / \sqrt{m}$ и $j$ на $m$.

Для среднего значения случайной величины $\chi_{m}$ в соответствии с равенством (4.9) имеем формулу

$$
\mathbf{M} \chi_{m}=\sum_{k=1}^{m-1} \frac{k(k+2)(m-1)_{k}}{(m+1)^{k+1}} .
$$

Эту формулу можно привести к виду

$$
\mathbf{M} \chi_{m}=\frac{(m-1) !}{(m+1)^{m}} \sum_{k=0}^{m-1} \frac{(m+1)^{k}}{k !}\left(m^{2}-1-(2 m-1) k+k(k-1)\right) .
$$

Используя преобразования, аналогичные примененным к равенству (3.15), отсюда получаем

$$
\mathbf{M} \chi_{m}=\frac{(m-1) !}{(m+1)^{m-1}} \sum_{k=0}^{m-1} \frac{(m+1)^{k}}{k !} .
$$

При $m \rightarrow \infty$ из равенства (4.11) с использованием формулы (1.9) получаем асимптотику

$$
\mathbf{M} \chi_{m}=\sqrt{\frac{\pi m}{2}}(1+o(1)) .
$$

$3^{0}$. Рассмотрим случайное отображение $\sigma \in F_{n}$, лес орграфа которого содержит заданную вершину. Обозначим через $\tilde{\chi}_{n}$ случайную величину, равную высоте заданной вершины. Распределение $\tilde{\chi}_{n}$ записывается в следующем виде:

$$
\mathbf{P}\left(\tilde{\chi}_{n}=k\right)=\frac{k+2}{V_{n}} \sum_{m=k+1}^{n}\left(\begin{array}{c}
n-1 \\
m-1
\end{array}\right)(n-m-1)^{n-m}(m-1)_{k}(m+1)^{m-k-2}, k=0,1, \ldots, n-1,
$$

где $V_{n}$ определяется формулой (3.2). 
Действительно, вероятность того, что орграф Г $\sigma)$ случайного отображения $\sigma \in F_{n}$ имеет лес с $m$ вершинами, среди которых находится заданная вершина, равна

$$
\frac{1}{V_{n}}\left(\begin{array}{c}
n-1 \\
m-1
\end{array}\right)(m+1)^{m-1}(n-m-1)^{n-m}
$$

Согласно (4.9) вероятность того, что заданная вершина в лесе с $m$ вершинами имеет высоту $k$, равна

$$
\frac{(k+2)(m-1)_{k}}{(m+1)^{k+1}}, k=0, \ldots, m-1 .
$$

Суммируя произведения этих вероятностей, приходим к формуле (4.13). Распределение вида (4.13) можно преобразовать к виду

$$
\mathbf{P}\left(\tilde{\chi}_{n}=k\right)=\frac{(k+2)(n-1)_{k}}{V_{n}} \underset{\sim}{\mathrm{H}}(n, k),
$$

где

$$
\underset{\sim}{\mathrm{H}}(n, k)=\sum_{m=0}^{n-k-1}\left(\begin{array}{c}
n-k-1 \\
m
\end{array}\right)(m+k+2)^{m-1}(n-k-m-2)^{n-k-m-1}, k=0,1, \ldots, n-1 .
$$

Применяя тождество Абеля (1.2), получаем

$$
\underset{\sim}{\mathrm{H}}(n, k)=A_{n-k-1}(k+2,-1,-1,0)=\frac{1}{k+2} n^{n-k-1} .
$$

Следовательно,

$$
\mathbf{P}\left(\tilde{\chi}_{n}=k\right)=\frac{(n-1)_{k} n^{n-k-1}}{V_{n}}, k=0,1, \ldots, n-1 .
$$

При $n \rightarrow \infty$ для всех $k=o\left(n^{2 / 3}\right)$ из формул (4.14) и (3.3) имеем

$$
\mathbf{P}\left(\tilde{\chi}_{n}=k\right)=\sqrt{\frac{2}{\pi}} e^{-\frac{k^{2}}{2 n}} \frac{1}{\sqrt{n}}(1+o(1)) .
$$

Из этой формулы вытекает предельная теорема.

Теорема 4. Пусть $u=\frac{k}{\sqrt{n}}$. Тогда при $n \rightarrow \infty$

$$
\begin{aligned}
& \mathbf{P}\left(\frac{\tilde{\chi}_{n}}{\sqrt{n}}=u\right)=\sqrt{\frac{2}{\pi}} e^{-\frac{u^{2}}{2}} \frac{1}{\sqrt{n}}(1+o(1)) \text { равномерно по } u=o\left(n^{1 / 6}\right), \\
& \mathbf{P}\left(\frac{\tilde{\chi}_{n}}{\sqrt{n}} \leq \alpha\right)=\sqrt{\frac{2}{\pi}} \int_{0}^{\alpha} e^{-\frac{x^{2}}{2}} d x(1+o(1)) .
\end{aligned}
$$


Предельное вероятностное распределение в (4.16) обычно называется отраженным нормальным распределением [6]. Для среднего значения случайной величины $\tilde{\chi}_{n}$ имеем формулу

$$
\mathbf{M} \tilde{\chi}_{n}=\frac{1}{V_{n}} \sum_{k=0}^{n-1} k(n-1)_{k} n^{n-k-1},
$$

из которой, используя формулу (3.2), находим, что

$$
\mathbf{M} \tilde{\chi}_{n}=\frac{n^{n}}{V_{n}}-1
$$

Отсюда при $\quad n \rightarrow \infty$ с учетом формулы (3.3) получаем

$$
\mathbf{M} \tilde{\chi}_{n}=\sqrt{\frac{2 n}{\pi}}(1+o(1))
$$

Для дисперсии случайной величины $\tilde{\chi}_{n}$ имеем следующее выражение:

$$
\mathbf{D} \tilde{\chi}_{n}=\frac{1}{V_{n}} \sum_{k=0}^{n-1} k^{2}(n-1)_{k} n^{n-k-1}-\left(\frac{n^{n}}{V_{n}}-1\right)^{2} .
$$

После преобразования суммы в выражении (4.18) и использования формулы (3.2) получаем

$$
\mathbf{D} \tilde{\chi}_{n}=n+1-\frac{2 n^{n}}{V_{n}}-\left(\frac{n^{n}}{V_{n}}-1\right)^{2} .
$$

Отсюда следует формула для дисперсии $\tilde{\chi}_{n}$

$$
\mathbf{D} \tilde{\chi}_{n}=n-\left(\frac{n^{n}}{V_{n}}\right)^{2},
$$

где $V_{n}$ определяется равенством (3.2).

При $n \rightarrow \infty$ из равенства (4.19) с учетом формулы (3.3) получаем асимптотику для дисперсии случайной величины $\tilde{\chi}_{n}$

$$
\mathbf{D} \tilde{\chi}_{n}=n\left(1-\frac{2}{\pi}\right)(1+o(1)) .
$$

Таким образом, дисперсия имеет порядок квадрата от среднего значения $\tilde{\chi}_{n}$. 


\section{Список литературы}

1. Harris B. Probability distributions related to random mappings. Ann. Math. Statist., 1960. V. 31. № 4. P. 1045-1062.

2. Сачков В.Н. Отображения конечного множества с ограничениями на контуры и высоту. - Теория вероятн. и ее примен., 1972. Т. 17. Вып. 4. C. 679-694.

3. Сачков В. Н. Комбинаторные методы дискретной математики. - М.: Наука, 1977.

4. Сачков В. Н. Вероятностные методы в комбинаторном анализе. М.: Наука, 1978.

5. Риордан Дж. Комбинаторные тождества. - М.: Наука, 1982.

6. Феллер $B$. Введение в теорию вероятностей и ее приложения. - М.: Мир, 1967.

7. Abel H. Oeuvres Completes. - Christiania C. Groendahe, 1839.

8. Колчин В. Ф. Случайные отображения. - М.: Наука, 1984. 
